\title{
Telomere Shortening linked to Disability and Mitochondrial DNA Copy Number in Patients with Relapsing-Remitting Mul- tiple Sclerosis
}

\author{
José Alfonso Cruz-Ramos ${ }^{1,7 *+}$, Gabriela del Carmen López-Armas ${ }^{2+}$, Eduardo Ignacio Díaz-Barba3 ${ }^{3}$, Mónica Nava- \\ rro-Meza ${ }^{4}$, Miguel Ángel Macías-Islas ${ }^{5}$, Ana Miriam Saldaña-Cruz ${ }^{6}$, Abraham Zepeda-Moreno7 , Héctor Raúl Pérez-

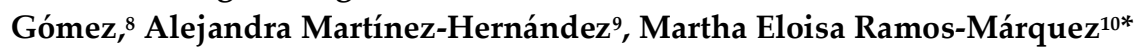 \\ 1 Instituto Jalisciense de Cancerología, Universidad de Guadalajara; jose.cruzr@academico.udg.mx \\ 2 Centro de Enseñanza Técnica Industrial. Plantel Colomos; glopez@ceti.mx \\ 3 Universidad de Guadalajara; eduardo.diaz@alumnos.udg.mx \\ 4 Centro Universitario del Sur. Universidad de Guadalajara; monica.navarro@cusur.udg.mx \\ 5 Departamento de Neurociencias. Centro Universitario de Ciencias de la Salud. Universidad de Guadalaja- \\ ra; miguelangelmacias@hotmail.com \\ 6 Departamento de Fisiología. Centro Universitario de Ciencias de la Salud; Universidad de Guadalajara; \\ ana.saldaña@academicos.udg.mx \\ 6 Departamento de Clínicas de la Reproducción humana, Crecimiento y Desarrollo Infantil. Centro Univer- \\ sitario de Ciencias de la Salud, Universidad de Guadalajara; abraham.zepeda@onkogenetik.com.mx \\ 7 Departamento de Clínicas Médicas. Centro Universitario de Ciencias de la Salud. Universidad de Guada- \\ laja-ra; jose.cruzr@academico.udg.mx , hectorraul.perez@cucs.udg.mx \\ 8 Doctorado de Farmacología. Centro Universitario de Ciencias de la Salud. Universidad de Guadalajara; \\ alegrije 01@hotmail.com \\ 9 Departamento de Biología Molecular y Genómica. Centro Universitario de Ciencias de la Salud. Universi- \\ dad de Guadalajara; eloisa10@yahoo.com \\ * Correspondence: jose.cruzr@academico.udg.mx (J.A.C.R); eloisa10@yahoo.com (M.E.R.M)
}

\begin{abstract}
Multiple sclerosis (MS) is a chronic autoimmune inflammatory disease that affects the nervous system. Peripheral blood leukocyte telomere length (LTL) and mitochondrial DNA copy number (mtDNA-CN) are potential biomarkers of disability and neurological damage. The present work evaluated LTL and mtDNA-CN in 75 relapsing-remittent MS (RRMS) patients 50 of whom had an Expanded Disability Status Scale (EDSS) 0 to 3 (mild-moderate disability), and 25 had an EDSS of 3.5 to 7 (severe disability). Absolute LTL and absolute mtDNA-CN were measured via realtime polymerase chain reaction (qPCR). The LTL and mtDNA-CN were significantly lower in RRMS severe disability than in RRMS mild-moderate disability $(3.924 \pm 0.124$ vs $2.854 \pm 0.092, \mathrm{p}<0.00001$; $75.14 \pm 1.77$ vs $68.06 \pm 1.608, \mathrm{p}<0.00001$, respectively). The LTL and mtDNA-CN showed a linear correlation in RRMS with mild-moderate disability $(\mathrm{r}=0.2986, \mathrm{p}=0.0351)$. In addition, in a binary logistic regression model the LTL can predict severe disabil-ity (AUC $=0.697, p=0.0031$, cutoff $\leq 3.0875$ $\mathrm{Kb}$, sensitivity $=73.1 \%$, specificity $=62.5 \%$ ), the prediction is improved by including age to the model (AUC $=0.765, \mathrm{p}<0.0001$, sensitivity=78.26\%, specificity=81.25\%). Aging is closely linked to the development of disability in RRMS and can be evaluated through LTL and mtDNA-CN absolute quantification..
\end{abstract}

Keywords: keyword 1; keyword 2; keyword 3 (List three to ten pertinent keywords specific to the article yet reasonably common within the subject discipline.)

\section{Introduction}

Multiple sclerosis (MS) is a chronic autoimmune disease of multifactorial origin (genetic susceptibility and environmental factors) with a heterogeneous clinical behavior featuring neurological dysfunction secondary to central and peripheral nervous system damage (1-3). Globally, MS is the leading non-traumatic cause of neuronal disability in 
young adults, affecting approximately 2.8 million people (4). Demyelination and axonal loss in the central nervous system (CNS) is crucial for the episodic and irreversible progression of MS, they are mediated by an inflammatory phase followed by a neurodegenerative one (5). At the onset, patients with MS may have mild psychological and cognitive impairments, which progress to severe neurological and motor limitations $(6,7)$. These neurodegenerative changes are assessed using the Expanded Disability Status Scale (EDSS) based on well-defined clinical and imaging parameters. Accordingly, patients are categorized from 0 (no signs, no symptoms) to 10 (death) (8-11). Patients with MS may have different phenotypes: primarily progressive multiple sclerosis (PPMS) manifests episodes of neurological dysfunction, is progressive and without recovery; relapsing-remitting multiple sclerosis (RRMS) characterized by episodes of neurological dysfunction with full or partial recovery, and finally, secondarily progressive multiple sclerosis (SPMS) results from an RRMS that acquires a PPMS-like pattern of progressive dysfunction without recovery (12). Neuroinflammation mechanisms in MS resemble those of aging but are seemingly activated early, exhibit greater intensity, and ultimately impact the course of the disease (13). Mitochondria and telomeres are closely linked to the aging process. Indeed, the core molecular mechanisms regulating aging are shared with the pathogenesis of most chronic degenerative diseases. Hence, the study of these common pathways is of current interest in MS research (14).

Telomeres are nucleoprotein structures that protect the ends of chromosomes, prevent deleterious structural changes such as intrachromosomal fusion, provide genomic stability, and regulate cellular senescence $(15,16)$. Recently, a systematic review found that telomere length (TL) is consistently shorter in MS patients than in healthy controls. This association is independent of age and correlates with greater disability, lower brain volume, higher recurrence rate, and shorter conversion time from recurrent to progressive MS (17).

Another important factor associated with cellular aging and neurodegeneration in MS is the mitochondrial deficit (18). The mitochondrial DNA copy number (mtDNA$\mathrm{CN}$ ) reliably estimates the number of mitochondria in the cell (19). Studies focused on mtDNA-CN in MS, both in cerebrospinal fluid and lymphocytes, showed a significant decrease in mtDNA-CN in patients compared to controls $(19,20)$. Furthermore, the AlKafaji study showed a significant decrease in mtDNA-CN in RRMS patients with more than ten years of diagnosis compared with those with less than ten years of diagnosis (19).

In the present study, we evaluated mtDNA-CN and leukocyte telomere length (LTL) as biomarkers to predict disability in patients with RRMS. Research insights into the link between aging and neurodegeneration can help classify MS patients according to prognosis and to design therapeutic strategies to prevent accelerated aging in MS.

\section{Materials and Methods}

\subsection{Design of the study}

The present research is a cross-sectional observational study. We enrolled 75 patients (50 women and 25 men) aged between 18 and 66 years old with RRMS without comorbidities (cancer, diabetes, hypertension, or other immunologic diseases), previously diagnosed, evaluated, and classified with EDSS by a neurologist. All patients fulfilled the McDonald diagnostic criteria (21). Group 1 (EDSS 0-3) with mild-moderate disability $(n=50)$ and group 2 (EDSS 3.5-7) with severe disability $(n=25)$. All patients were recruited from the Institute of Experimental Clinical Therapy (INTEC) MS cohort, University Center of Health Sciences (CUCS) of the University of Guadalajara (Figure 1). In addition, age, time after disease onset, disease progression index and actual treatment were recorded in both groups. This study complies with the Declaration of Helsinki guidelines. 
The ethics committee of the CUCS approved the study with the number CI-03519. All participants involved in the study signed informed consent.

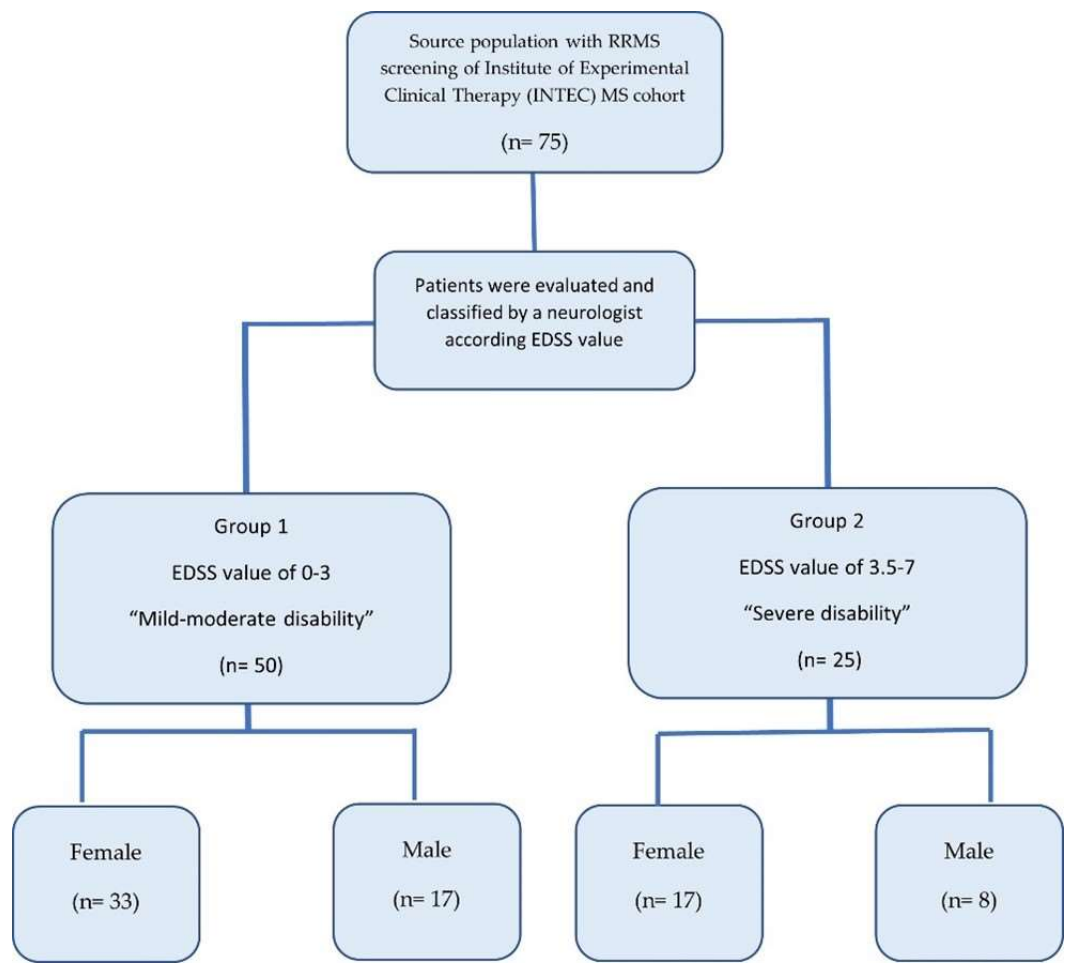

Figure 1. Flowchart of patient selection.

\subsection{Absolute quantification of telomere length and mtDNA copy number}

Total venous blood samples were collected for separation of leukocyte and DNA extraction by Miller method, once obtained were stored at $-20{ }^{\circ} \mathrm{C}$ until analysis (39). The quantification of mtDNA-CN and LTL was assessed by the commercial assay kit Absolute Human Telomere Length and Mitochondrial DNA Copy Number Dual Quantification qPCR (ScienCell Research Laboratories, San Diego, CA, USA) according to the manufacturer's protocol. For PCR reaction (per sample): $10 \mu \mathrm{L}$ of $2 X$ GoldNStart TaqGreen qPCR master mix, $2 \mu \mathrm{L}$ of primer solution (Tel, mtDNA or SCR), $7 \mu \mathrm{L}$ of nuclease-free water, and $1 \mu \mathrm{L}$ of DNA problem ( $5 \mathrm{ng}$ ). Initial denaturation at $95^{\circ} \mathrm{C}$ for $10 \mathrm{~min}$, followed by 32 cycles with denaturation $\left(95^{\circ} \mathrm{C}\right.$ for $\left.20 \mathrm{sec}\right)$, hybridization $\left(52^{\circ} \mathrm{C}\right.$ for $\left.20 \mathrm{sec}\right)$, and extension $\left(72^{\circ} \mathrm{C}\right.$ for $\left.45 \mathrm{sec}\right)$. As a constituent region, a region of $100 \mathrm{bp}$ length is used on chromosome 17, which is recognized by the first set of SCR (single copy reference). A reference DNA with a known concentration (telomere length $348 \pm 11 \mathrm{~kb}$ per diploid cell and $\mathrm{mt}$ DNA of $1.27 \pm 0.03 \times 103$ copies per diploid cell) was used.

\subsection{Statistical analysis}

The statistical analysis consisted of descriptive statistics (means, frequencies and percentages) to describe the groups of patients. The Anderson-Darling $(n>30)$ or D'Agostino-Pearson $(n<30)$ tests were used to evaluate the normality of the data. The outliners were identified with the Rout method. For the comparison between the groups of patients with mild-moderate disability vs. severe disability, inferential statistics were 
used with parametric tests such as Student's T for variables with normal distribution; Mann's U test was used for variables with non-normal distribution; Chi square test or Fisher's exact test was used for the comparison of frequencies. The predictive capacity (sensitivity, specificity, PPV and NPV) was evaluated with ROC curves. To predict severe disability, binary logistic regression models were developed, which were evaluated with the Akaike corrected information criterion and the $\mathrm{Z}$ statistic for comparison of ROC curves (LTL vs. Age vs. LTL + Age). All analysis were performed using GraphPad Prism version 9.02 for Windows (La Jolla California USA, www.graphpad.com) and MedCalc Statistical Software version 15.8 (Ostend, Belgium; https://www.medcalc.org).

\section{Results}

\subsection{Patients}

Seventy-five patients with RRMS, one group of 50 RRMS with mild to moderate disability (EDSS from 0 to 3), and the other group of 25 RRMS patients with severe disability (EDSS from 3.5 to 7 ), were enrolled. Of the total number of patients, there were 50 women and 25 men. The mean age was $39.48 \pm 11.52$, mean years since diagnosis was $8.607( \pm 5.881)$, mean progression rate was $0.6726 \pm 0.8374$, and the mean EDSS $2.907 \pm 1.712$. There was no significant difference regarding sex, age, or time since diagnosis between the 2 study groups. There was significant difference regarding progression rate and EDSS. The EDSS of the RRMS with mild disability group (EDSS 0 to 3 ) was $1.91 \pm 0.8846$ and the EDSS of the RRMS with advanced disability group (EDSS 3.5 to 7 ) was $4.9 \pm 1.109$ (Table 1).

Table 1. Characteristics of groups of patients and their pharmacological treatments. Group 1 with mild to moderate disability (EDSS 0-3) and group 2 with severe disability (EDSS 3.5-7).

\begin{tabular}{lcc}
\hline Characteristic & $\begin{array}{c}\text { Group 1, } \mathbf{n = 5 0} \\
\text { "Mild-moderate disability" }\end{array}$ & $\begin{array}{c}\text { Group 2, } \mathbf{n}=\mathbf{2 5} \\
\text { “Severe disability" }\end{array}$ \\
\hline Female & $33(66 \%)$ & $17(68 \%)$ \\
Male & $17(34 \%)$ & $8(32 \%)$ \\
Age & $37.70( \pm 11.409)$ & $43.04( \pm 11.110)$ \\
Years since diagnosis & $7.78( \pm 5.1211)$ & $10.26( \pm 6.9868)$ \\
EDSS* & $1.91( \pm 0.8846)$ & $4.9( \pm 1.109)$ \\
Progression rate* & $0.5139( \pm 0.6355)$ & $0.9898( \pm 1.0864)$ \\
Treatment: & & \\
Glatiramer Acetate & $19(38 \%)$ & $9(36 \%)$ \\
Rituximab & $4(8 \%)$ & $3(12 \%)$ \\
Interferon $\beta$ & $14(28 \%)$ & $5(2 \%)$ \\
Fingolimod & $5(10 \%)$ & $2(8 \%)$ \\
Azathioprine & 0 & $3(12 \%)$ \\
Natalizumab & $1(2 \%)$ & $1(4 \%)$ \\
Dimethyl fumarate & $3(6 \%)$ & $1(4 \%)$ \\
None & $4(8 \%)$ & $1(4 \%)$ \\
\hline
\end{tabular}

EDSS=Expanded Disability Status Scale

Progression rate $=\mathrm{EDSS} / \mathrm{years}$ with the disease

${ }^{*} \mathrm{p}<0.05$. Progression rate comparison by student $\mathrm{T}$ test and EDSS by Mann Whitney test.

2.2 Absolute LTL and mtDNA-CN in mild-moderate disability vs. severe disability 
Comparisons of LTL $(3.924 \pm 0.124$ vs. $2.854 \pm 0.090)$ and mtDNA-CN $(75.14 \pm 1.77$ vs. $68.06 \pm 1.60$ ) between the groups with mild-moderate disability and severe disability were significant (Figure 2).

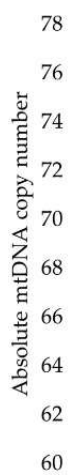

a

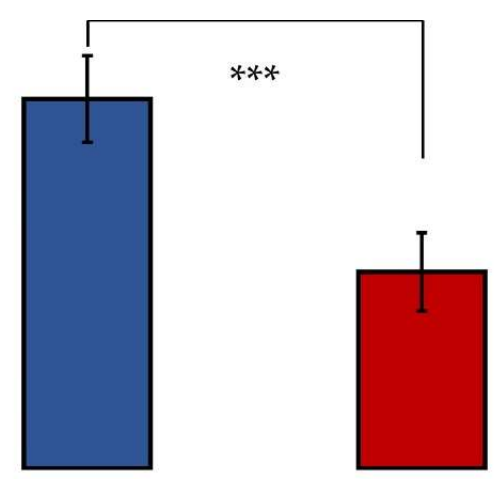

EDSS 0-3

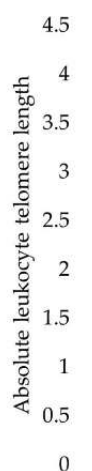

$\mathrm{b}$

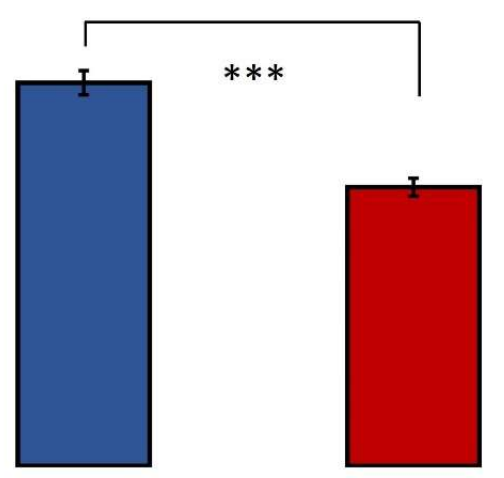

EDSS 0-3

EDSS 3.5-7

Figure 2. (a) Absolute mitochondrial DNA copy number (mtDNA-CN) from relapsing-remitting multiple sclerosis (RRMS) patients with mild-moderate disability (blue) and severe disability (red). (b) Absolute leukocyte telomere length (LTL) in RRMS patients with mild disability (blue) and severe disability (red). ${ }^{* * *}=p<0.00001$, unpaired student T-test, normality evaluated by D'Agostino \& Pearson test.

\subsection{Correlation between LTL and mtDNA-CN}

Pearson's correlation test was significant in the mild-moderate disability group (Figure 3). There was no correlation in the severe disability group.

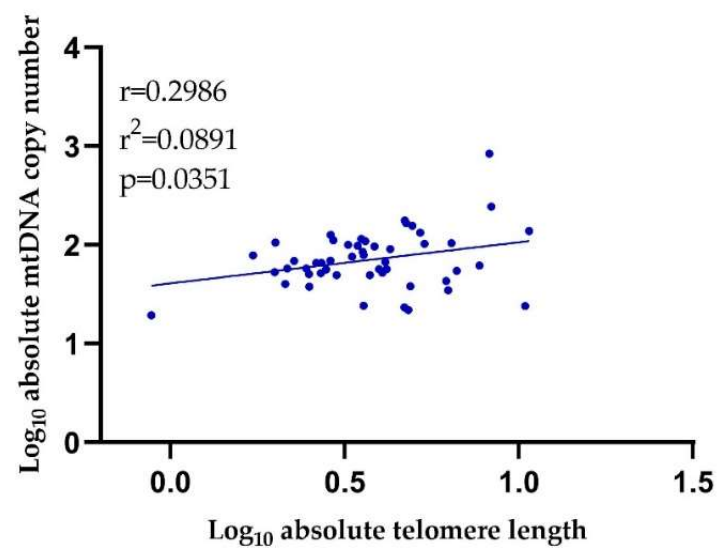

Figure 3. Correlation (Pearson) between $\log _{10}$ LTL and $\log _{10}$ mtDNA-CN in RRMS patients with mild-moderate disability.

\subsection{Telomere Disability Prediction}

The predictive capacity of LTL was evaluated directly (raw data), the receiveroperating characteristic (ROC) curves was significant $(\mathrm{p}=0.003)$, and the area under the curve (AUC) was 0.697. The cut-off point was obtained through the Youden index, 
and the associated criterion was $\leq 3.0875 \mathrm{~Kb}$, which showed a sensitivity of $73.1 \%$ and specificity of $62.5 \%$, positive predictive value (PPV) of $48.6 \%$, and negative predictive value (NPV) of $83.3 \%$ (Figure 4 ).

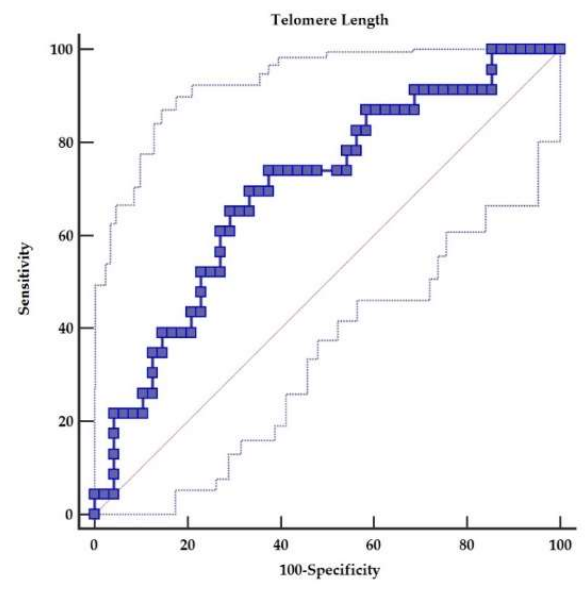

Figure 4. Receiver-operating characteristic (ROC) curves for LTL (raw data) in patients with RRMS.

\subsection{Binary Logistic Regression Model of LTL and Age}

Predictive models based on binary logistic regression was developed. Of the variables introduced, only LTL maintains significance when controlling for variables in Wald test (Table 2). However, the integration of age together with LTL improved the precision of the model, which did not occur with the rest of the variables.

Table 2. Features evaluated in the binary logistic regression model for the prediction of severe disability in MS.

\begin{tabular}{lccc}
\hline Variable & Odds ratio & $\mathbf{9 5 \%}$ CI & p \\
\hline LTL* & 0.5855 & 0.3672 to 0.9336 & 0.0245 \\
\hline mtDNA-CN & 0.9980 & 0.9810 to 1.0152 & 0.8142 \\
\hline Age & 1.0331 & 0.9789 to 1.0903 & 0.2362 \\
\hline Sex (female) & 1.0040 & 0.3057 to 3.2974 & 0.8143 \\
\hline Years since diagnosis & 1.0585 & 0.9572 to 1.1706 & 0.2678 \\
\hline
\end{tabular}

LTL: Leukocyte telomere length; mtDNA-CN: mitochondrial DNA copy number.

* $\mathrm{p}<0.05$, Wald test. $\mathrm{CI}=$ confidence interval

LTL and age had the highest precision and was the most appropriate according to Akaike's criteria $(\mathrm{AUC}=0.765, \mathrm{p}<0.0001$, sensitivity $=78.26 \%$, specificity $=81.25 \%$, PPV $=66.7$ and NPV $=88.6 \%$ ) (Figure 5). Pairwise comparison of ROC curves, between LTL and age versus LTL did not reach significance $(\mathrm{p}=0.1429)$, so the equivalence of the models (LTL and age vs. LTL alone) was not ruled out (Figure 6). 


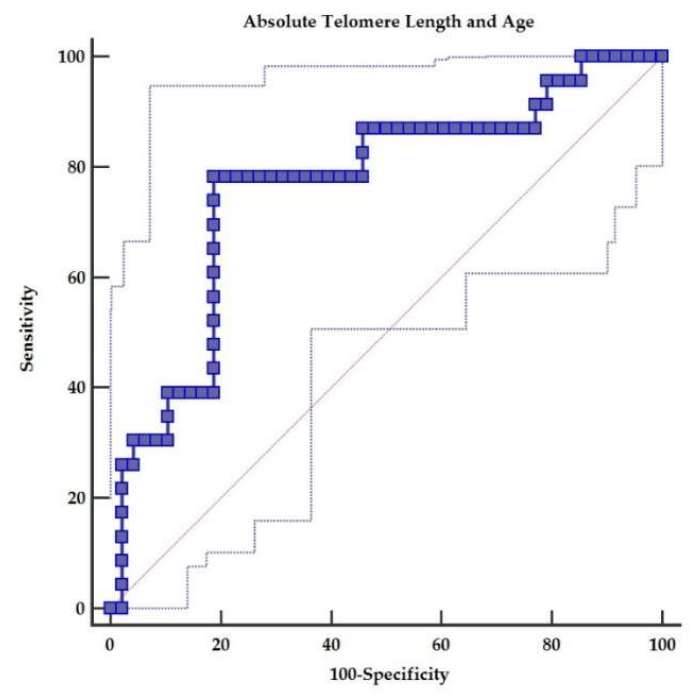

Figure 5. Receiver-operating characteristic (ROC) curve for binary logistic regression model for LTL and age in patients with RRMS.

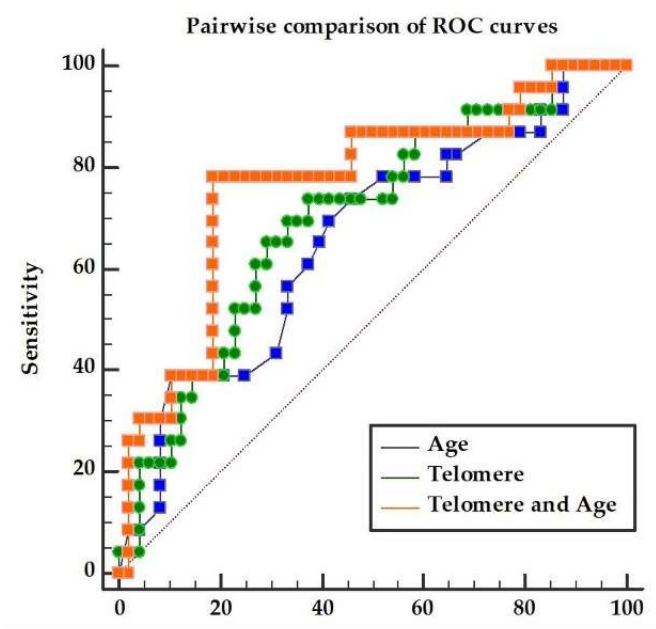

\begin{tabular}{lccc}
$\begin{array}{l}\text { Univariate analysis of Age and LTL (raw data); Logistic Regression of LTL and age; } \\
\text { pairwise comparison between models. }\end{array}$ & AUC & $95 \%$ CI & p value \\
\hline Variable & 0.658 & 0.536 to 0.767 & 0.0488 \\
Age & 0.697 & 0.577 to 0.801 & 0.0031 \\
LTL & 0.765 & 0.650 to 0.858 & 0.0001 \\
LTL and Age & & & \\
$\begin{array}{l}\text { Pairwise comparison of ROC } \\
\text { curves }\end{array}$ & & 0.000682 to 0.214 & 0.0485 \\
Age $v$ s. Age+LTL & & -0.0230 to 0.159 & 0.1429 \\
LTL $v$ s. Age+LTL & -0.140 to 0.219 & 0.6673 \\
Age $v$ s. LTL & & & \\
\hline
\end{tabular}

LTL: Leukocyte Telomere Length AUC: Area under the ROC curve

Figure 6. Pairwise comparison of ROC curves of LTL (raw data), age (raw data) and LTL and age (binary logistic regression).

\section{Discussion}

In recent years, MS has been approached from the perspective of biological aging as the trigger of the cellular and immunological changes involved in the development of neuroinflammation, neurodegeneration, and disability. Several authors consider that, among the multiple factors underlying neurodegeneration, accelerated neurological aging occurs in MS. Telomeres are found at the ends of chromosomes and are made up of repeats of the hexanucleotide TTAGGG, a non-coding sequence $(15,22)$. Telomere changes contribute to the pathogenesis of several multifactorial chronic diseases, including neurological disorders. Loss of chromosomal integrity due to shorten telomeres facilitates fusions with other chromosomes, mutations, and other events that impair cell function, decrease cell division, and lead to cell aging $(23,24)$. 
Mitochondria is an organelle that regulate intracellular calcium homeostasis, ATP generation, programmed cell death (apoptosis), reactive oxygen species (ROS) production, and aging processes, all of which affect the telomere structure and function (25). Numerical and structural alterations of mitochondria is pathogenic, especially in high-energy-demanding tissues such as muscle and the nervous system(26). Age and accelerated aging are factors of neurological deterioration in MS. Age is considered a strong independent factor to develop a progressive MS phenotype via complex and multidirectional interactions with aging and degenerative processes $(27,28)$. In this sense, the telomeres and mitochondria are two of the most important cellular components in the regulation of aging and disease.

The patients enrolled in this study were primarily young (mean $39.48 \pm 11.52$ years) and selected in such a way that the distribution of MS was similar in both sexes and to the proportion of mild-moderate/severe disability observed in the general population; hence, the effect of sex and age on LTL or mtDNA-CN was minimized. The wide variability of LTL and mtDNA-CN reflects the interactions of organisms with environmental factors and the influence of endogenous factors (genes, hormones, ROS, among others); notably, up to $70 \%$ of the variance of TL is explained by heritability $(29,30)$.

Although the variability in TL is determined by multiple factors, in the group with a mild-moderate disability, we found a low but significant linear correlation $(r=0.2986$, $\mathrm{p}=0.0351$ ) between LTL and mtDNA-CN. Even if a similar finding was previously documented in elderly women (31), our study is the first to correlate these two senescence parameters in patients with MS. The lack of such a correlation in the severe disability group suggests that this correlation was lost in advanced disability stages due to greater heterogeneity in the processes of aging and neurodegeneration, as well as by the influence of other factors such as increased immune activation, chronic inflammation, and age $(13,32)$. The bidirectional interaction between mitochondria and TL result from different mechanisms related to mitochondrial function, such as oxidative stress, apoptosis, energy efficiency of the respiratory chain, and chronic inflammation. Of note, all of these can produce telomere shortening and subsequent exposure of subtelomeric DNA, which in turn affect mitochondrial function through activation of genes that prevent genetic damage such as p53 and p21(33). The aging is fundamental for the progression of disability; actually, senescent changes have been identified in multiple cell lineages of the nervous system that are affected during the evolution of MS, such as neurons, microglia, oligodendrocytes, and astrocytes (34-38). In addition, we compared the absolute quantification of LTL and mtDNA-CN in mild-moderate disability patients versus patients with severe disability and found significant differences for both parameters $(3.924 \pm 0.124$ vs $2.854 \pm 0.092, \mathrm{p}<0.00001 ; 75.14 \pm 1.77$ vs $68.06 \pm 1.608, \mathrm{p}<0.00001$, respectively); thus, such discrepancies support the interdependence of LTL and mtDNA-CN in the different phases of disability in MS.

Telomere biology and age play a crucial role in health and disease. In several studies, age has been reported as one of the most important factors for conversion of a RRMS into SPMS (39-41). This clinical distinction reflects the underlying neurological damage: in the former phenotype the damage is predominantly inflammatory whereas in the progressive phenotype it is neurodegenerative $(5,42)$. Moreover, LTL can serve as a biomarker of immunosenescence and has been linked to the progression of neurological damage (40); thereby, LTL could be useful for assessing and predicting disability in MS(43). We have found LTL to be an independent predictor of disability. After integrating possible predictors (LTL, mtDNA-CN, sex, age, and time with the disease) to the binary logistic regression model, only age and LTL were useful for prediction. The ROC curve for direct LTL values alone has moderate predictive capability $(\mathrm{AUC}=0.697, \mathrm{p}=0.003$, cutoff $\leq 3.0875 \mathrm{~Kb}$, sensitivity $=73.1 \%$, specificity $=62.5 \%, \mathrm{PPV}=48.6 \%$, NPV $=83.3 \%$ ). The high NPV of LTL 
identifies LTL as a relevant biomarker for assessing disability status. Interestingly, when LTL and age were combined in the binary logistic regression model, accuracy increased (AUC: $0.765, \mathrm{p}<0.001$, sensitivity $=78.26 \%$, specificity $=81.25 \%$, PPV $=66.7 \%, \mathrm{NPV}=$ $88.6 \%$ ). Although LTL and age are related risk factors that predict disability, LTL remains as a disability factor even when analyzed independently from other variables. This is of great importance since age is the highest prognostic factor for MS progression (44); however, LTL can be as strong or even stronger than age as a predictor of disability status and/or disease progression in MS.

\section{Conclusions}

More studies, especially longitudinal ones with large samples that fully address the different MS phenotypes, are required to establish the clinical utility of LTL for the evaluation and prediction of disability, as well as to unravel the molecular mechanisms involved in the process of neuroinflammation, neurodegeneration, and aging and its relationship with the structure and function of telomeres and mitochondria in MS.

Supplementary Materials: Database is available online at https://figshare.com/articles/dataset/TLT_mtDNA_MS/17097470

Author Contributions: Conceptualization of this research was made by J.A.C.R; G.C.L.A and M.E.R.M; methodology was developed by E.I.D.B; validation of qPCR was supported by A.Z.M and M.N.M; formal analysis, J.A.C.R.; investigation, M.A.M.I and A.M.S.C; investigation and sample collection, A.M.H; project administration and resources, H.R.P.G.; data curation, J.A.C.R and E.I.D.B.; writing - original draft preparation, J.A.C.R; G.C.L.A and M.E.R.M; writing-review and editing, J.A.C.R; G.C.L.A, and M.E.R.M visualization, A.M.S.C.; supervision, M.A.M.I.; project administration, H.R.P.G.; funding acquisition, J.A.C.R. All authors have read and agreed to the published version of the manuscript.

Funding: This research was supported by PROSNI 2021 of the University of Guadalajara.

Institutional Review Board Statement: The study was conducted according to the guidelines of the Declaration of Helsinki and approved by the Ethics Committee and Biosafety Committee from Centro Universitario de Ciencias de la Salud (CUCS) Universidad de Guadalajara, Jalisco, Mexico, with registration number CI-03519.

Informed Consent Statement: Informed consent was obtained from all subjects involved in the study.

Data Availability Statement: Details regarding where data supporting reported results can be requested at the following e-mail address: jose.cruzr@academico.udg.mx

Acknowledgments: We are deeply grateful to Dr. Horacio Rivera Ramirez for their help in manuscript editing.

Conflicts of Interest: The authors declare no conflict of interest.

\section{References}

1. Goodin DS. The causal cascade to multiple sclerosis: a model for MS pathogenesis. Scalas E, editor. PLoS One. 2009 Feb;4(2):e4565.

2. Goodin DS. The epidemiology of multiple sclerosis : insights to disease pathogenesis. In: Goodi DS, editor. Multiple Sclerosis and Related Disorders. Elsevier B.V.; 2014. p. 231-66. 
3. Goodin DS. The epidemiology of multiple sclerosis: insights to a causal cascade. Handb Clin Neurol [Internet]. 2016 [cited 2021 Nov 8];138:173-206. Available from: https://pubmed.ncbi.nlm.nih.gov/27637959/

4. Walton C, King R, Rechtman L, Kaye W, Leray E, Marrie RA, et al. Rising prevalence of multiple sclerosis worldwide: Insights from the Atlas of MS, third edition. Mult Scler [Internet]. 2020 Dec 1 [cited 2021 Nov 21];26(14):1816-21. Available from: https://pubmed.ncbi.nlm.nih.gov/33174475/

5. Reich DS, Lucchinetti CF, Calabresi PA. Multiple sclerosis. N Engl J Med. 2018;378(2):169-80.

6. Confavreux C, Vukusic S. Natural history of multiple sclerosis: A unifying concept. Brain. 2006;129(3):606-16.

7. Islas MÁM, Ciampi E. Assessment and Impact of Cognitive Impairment in Multiple Sclerosis: An Overview. Biomedicines [Internet]. 2019 Mar 1 [cited 2021 Dec 6];7(1). Available from: /pmc/articles/PMC6466345/

8. Cadavid D, Tang Y, O'Neill G. Responsiveness of the Expanded Disability Status Scale (EDSS) to disease progression and therapeutic intervention in progressive forms of multiple sclerosis. Rev Neurol. 2010 Sep;51(6):321-9.

9. Kurtzke JF. Rating neurologic impairment in multiple sclerosis: an expanded disability status scale (EDSS). Neurology [Internet]. 1983 [cited 2021 Nov 8];33(11):1444-52. Available from: https://pubmed.ncbi.nlm.nih.gov/6685237/

10. Kurtzke JF. On the origin of EDSS. Mult Scler Relat Disord [Internet]. 2015 Mar 1 [cited 2021 Nov 8];4(2):95-103. Available from: https://pubmed.ncbi.nlm.nih.gov/25787185/

11. Şen S. Neurostatus and EDSS Calculation with Cases. Arch Neuropsychiatry. 2018;55(Supplent 1):S80-3.

12. Lublin FD, Reingold SC, Cohen JA, Cutter GR, Sørensen PS, Thompson AJ, et al. Defining the clinical course of multiple sclerosis: The 2013 revisions. Neurology [Internet]. 2014 Jul 15 [cited 2021 Nov 21];83(3):278. Available from:/pmc/articles/PMC4117366/ 13. Musella A, Gentile A, Rizzo FR, Vito F De, Fresegna D, Bullitta S, et al. Interplay between age and neuroinflammation in multiple sclerosis: Effects on motor and cognitive functions. Front Aging Neurosci [Internet]. 2018 Aug 8 [cited 2021 Nov 23];10(AUG). Available from: /record/2018-41142-001

14. Patergnani S, Fossati V, Bonora M, Giorgi C, Marchi S, Missiroli S, et al. Mitochondria in Multiple Sclerosis: Molecular Mechanisms of Pathogenesis. Int Rev Cell Mol Biol. 2017 Jan 1;328:49-103.

15. Saretzki G. Telomeres, Telomerase and Ageing. Subcell Biochem [Internet]. 2018 [cited 2021 Nov 16];90:221-308. Available from: https://pubmed.ncbi.nlm.nih.gov/30779012/

16. Moyzis RK, Buckingham JM, Cram LS, Dani M, Deaven LL, Jones MD, et al. A highly conserved repetitive DNA sequence, (TTAGGG)n, present at the telomeres of human chromosomes. Proc Natl Acad Sci U S A [Internet]. 1988 [cited 2021 Nov 21];85(18):6622-6. Available from: https://pubmed.ncbi.nlm.nih.gov/3413114/

17. Bühring J, Hecker M, Fitzner B, Zettl UK. Systematic review of studies on telomere length in patients with multiple sclerosis. Aging Dis. 2021;12(5):1272-86.

18. Mao P, Reddy PH. Is multiple sclerosis a mitochondrial disease? Biochim Biophys Acta Mol Basis Dis [Internet]. 2010 Jan [cited 2021 Nov 21];1802(1):66. Available from: /pmc/articles/PMC2790545/

19. Al-Kafaji G, Bakheit HF, Alharbi MA, Farahat AA, Jailani M, Ebrahin BH, et al. Mitochondrial DNA Copy Number in Peripheral Blood as a Potential Non-invasive Biomarker for Multiple Sclerosis. NeuroMolecular Med. 2020;22(2):304-13.

20. Lowes H, Pyle A, Duddy M, Hudson G. Cell-free mitochondrial DNA in progressive multiple sclerosis. Mitochondrion. 2018;46:307-12.

21. Thompson AJ, Banwell BL, Barkhof F, Carroll WM, Coetzee T, Comi G, et al. Diagnosis of multiple sclerosis: 2017 revisions of the McDonald criteria. Lancet Neurol [Internet]. 2018 Feb 1 [cited 2021 Dec 6];17(2):162-73. Available from: http://www.thelancet.com/article/S1474442217304702/fulltext

22. Moyzis RK, Buckingham JM, Cram LS, Dani M, Deaven LL, Jones MD, et al. A highly conserved repetitive DNA sequence, (TTAGGG)n, present at the telomeres of human chromosomes. Proc Natl Acad Sci U S A [Internet]. 1988 [cited 2021 Nov 16];85(18):6622-6. Available from: https://pubmed.ncbi.nlm.nih.gov/3413114/

23. Anitha A, Thanseem I, Vasu MM, Viswambharan V, Poovathinal SA. Telomeres in neurological disorders. Adv Clin Chem [Internet]. 2019 Jan 1 [cited 2021 Nov 8];90:81-132. Available from: https://pubmed.ncbi.nlm.nih.gov/31122612/

24. Wang S, Madu CO, Lu Y. Telomere and Its Role in Diseases. Oncomedicine. 2019 Feb 8;4:1-9.

25. Zheng Q, Huang J, Wang G. Mitochondria, Telomeres and Telomerase Subunits. Front Cell Dev Biol. 2019;7(November):1-10.

26. Lezi E, Swerdlow RH. Mitochondria in Neurodegeneration. Adv Exp Med Biol [Internet]. 2012 [cited 2021 Nov 16];942:269. Available from: /pmc/articles/PMC3618469/

27. Scalfari A, Neuhaus A, Daumer M, Ebers GC, Muraro PA. Age and disability accumulation in multiple sclerosis. Neurology [Internet]. 2011 Sep 27 [cited 2021 Nov 10];77(13):1246-52. Available from: https://pubmed.ncbi.nlm.nih.gov/21917763/

28. Sanai SA, Saini V, Benedict RHB, Zivadinov R, Teter BE, Ramanathan M, et al. Aging and multiple sclerosis. Mult Scler [Internet]. 2016 May 1 [cited 2021 Nov 23];22(6):717-25. Available from: https://pubmed.ncbi.nlm.nih.gov/26895718/

29. Hjelmborg JB, Dalgård C, Möller S, Steenstrup T, Kimura M, Christensen K, et al. The heritability of leucocyte telomere length dynamics. J Med Genet [Internet]. 2015 [cited 2021 Nov 22];52(5):297-302. Available from: https://pubmed.ncbi.nlm.nih.gov/25770094/

30. Broer L, Codd V, Nyholt DR, Deelen J, Mangino M, Willemsen G, et al. Meta-analysis of telomere length in 19713 subjects reveals high heritability, stronger maternal inheritance and a paternal age effect. Eur J Hum Genet [Internet]. 2013 Oct [cited 2021 Nov 22];21(10):1163. Available from: /pmc/articles/PMC3778341/

31. Kim JH, Kim HK, Ko JH, Bang H, Lee DC. The Relationship between Leukocyte Mitochondrial DNA Copy Number and Telomere Length in Community-Dwelling Elderly Women. PLoS One [Internet]. 2013 Jun 13 [cited 2021 Nov 23];8(6):e67227. Available from: https://journals.plos.org/plosone/article?id=10.1371/journal.pone.0067227 
32. Kordinas V, Ioannidis A, Chatzipanagiotou S. The Telomere/Telomerase System in Chronic Inflammatory Diseases. Cause or Effect? Genes (Basel) [Internet]. 2016 Sep 3 [cited 2021 Nov 23];7(9). Available from: /pmc/articles/PMC5042391/

33. Passos JF, Saretzki G, Ahmed S, Nelson G, Richter T, Peters H, et al. Mitochondrial dysfunction accounts for the stochastic heterogeneity in telomere-dependent senescence. PLoS Biol [Internet]. 2007 May [cited 2021 Nov 16];5(5):1138-51. Available from: https://pubmed.ncbi.nlm.nih.gov/17472436/

34. Pertusa M, García-Matas S, Rodríguez-Farré E, Sanfeliu C, Cristòfol R. Astrocytes aged in vitro show a decreased neuroprotective capacity. J Neurochem [Internet]. 2007 May [cited 2021 Nov 16];101(3):794-805. Available from: https://pubmed.ncbi.nlm.nih.gov/17250685/

35. Mansour H, Chamberlain CG, Weible MW, Hughes S, Chu Y, Chan-Ling T. Aging-related changes in astrocytes in the rat retina: imbalance between cell proliferation and cell death reduces astrocyte availability. Aging Cell [Internet]. 2008 Aug [cited 2021 Nov 16];7(4):526-40. Available from: https://pubmed.ncbi.nlm.nih.gov/18489730/

36. Al-Mashhadi S, Simpson JE, Heath PR, Dickman M, Forster G, Matthews FE, et al. Oxidative Glial Cell Damage Associated with White Matter Lesions in the Aging Human Brain. Brain Pathol [Internet]. 2015 Sep 1 [cited 2021 Nov 16];25(5):565-74. Available from: https://pubmed.ncbi.nlm.nih.gov/25311358/

37. Jurk D, Wang C, Miwa S, Maddick M, Korolchuk V, Tsolou A, et al. Postmitotic neurons develop a p21-dependent senescencelike phenotype driven by a DNA damage response. Aging Cell [Internet]. 2012 Dec [cited 2021 Nov 16];11(6):996-1004. Available from: https://pubmed.ncbi.nlm.nih.gov/22882466/

38. Martínez-Cué C, Rueda N. Cellular Senescence in Neurodegenerative Diseases. Front Cell Neurosci. 2020 Feb 11;14:16.

39. Gray V, Arnett P. Aging with multiple sclerosis: cognitive, emotional and neuropathological considerations. Neurodegener Dis Manag. 2014;4(2):187-94.

40. Miner AE, Graves JS. What telomeres teach us about MS. Mult Scler Relat Disord. 2021;54:103084.

41. Leray E, Yaouanq J, Le Page E, Coustans M, Laplaud D, Oger J, et al. Evidence for a two-stage disability progression in multiple sclerosis. Brain. 2010/04/27. 2010 Jul;133(Pt 7):1900-13.

42. Cree BAC, Reich DE, Khan O, De Jager PL, Nakashima I, Takahashi T, et al. Modification of Multiple Sclerosis Phenotypes by African Ancestry at HLA. Arch Neurol. 2009 Feb;66(2):226-33.

43. Krysko KM, Henry RG, Cree BAC, Lin J, Caillier S, Santaniello A, et al. Telomere Length Is Associated with Disability Progression in Multiple Sclerosis. Ann Neurol. 2019;86(5):671-82.

44. Ghione E, Bergsland N, Dwyer MG, Hagemeier J, Jakimovski D, Paunkoski I, et al. Aging and Brain Atrophy in Multiple Sclerosis. J Neuroimaging. 2019;29(4):527-35. 